Article

\title{
Anti-Sweep Jamming Design and Implementation Using Multi-Channel Harmonic Timing Sequence Detection for Short-Range FMCW Proximity Sensors
}

\author{
Zhijie Kong, Ping Li, Xiaopeng Yan and Xinhong Hao * \\ School of Mechatronical Engineering, Beijing Institute of Technology, Beijing 100081, China; \\ ethankong@163.com (Z.K.); liping85@bit.edu.cn (P.L.); yanxiaopeng@bit.edu.cn (X.Y.) \\ * Correspondence: haoxinhong@bit.edu.cn; Tel.: +86-010-6891-4850
}

Received: 21 July 2017; Accepted: 5 September 2017; Published: 6 September 2017

\begin{abstract}
Currently, frequency-modulated continuous-wave (FMCW) proximity sensors are widely used. However, they suffer from a serious sweep jamming problem, which significantly reduces the ranging performance. To improve its anti-jamming capability, this paper analyzed the response mechanism of a proximity sensor with the existence of real target echo signals and sweep jamming, respectively. Then, a multi-channel harmonic timing sequence detection method, using the spectrum components' distribution difference between the real echo signals and sweep jamming, is proposed. Moreover, a novel fast Fourier transform (FFT)-based implementation was employed to extract multi-channel harmonic information. Compared with the traditional band-pass filter (BPF) implementation, this novel realization scheme only computes FFT once, in each transmission cycle, which significantly reduced hardware resource consumption and improved the real-time performance of the proximity sensors. The proposed method was implemented and proved to be feasible through the numerical simulations and prototype experiments. The results showed that the proximity sensor utilizing the proposed method had better anti-sweep jamming capability and ranging performance.
\end{abstract}

Keywords: FMCW; proximity sensor; anti-sweep jamming; timing sequence detection; ranging

\section{Introduction}

Frequency-modulated continuous wave (FMCW) sensors constantly transmit and receive signals and is thus capable of maintaining a high signal-to-noise ratio with much less peak power than a corresponding pulse sensor system. Due to its simple structure, the FMCW sensors are widely used in a plurality of areas [1], such as healthcare [2], security [3], navigation [4], vehicle collision warning system [5], geodesy [6], helicopter and aircraft landing control [7], and other related areas. The ranging performance is a key parameter for the FMCW sensor. However, it is significantly affected by unintended interference [8] and intended jamming [9]. Among them, the intended jamming has become a serious threat to FMCW sensors, especially sweep jamming $[10,11]$, which combines the properties of both spot jamming and barrage jamming by rapidly sweeping a narrow band of jamming signals over a wide frequency band [12]. Hence, many previous studies have focused on increasing the anti-sweep jamming capability of short-range proximity sensors.

Prior studies to increase the anti-sweep jamming capability of FMCW sensors can be categorized into two categories: transmitted waveform design [5,13-15] and beat signal processing [16-19]. Reference [5] designed a new transceiver and utilized the fractional Fourier transform (FRFT), which was sensitive to the FM slope, to suppress jamming. Perrin used the spectrum characteristics of echoes to detect targets [16], while Xiao et al. [17] distinguished the target position according to the Doppler characteristics of different encounters process. Based on an analysis of difference between the target 
echoes and jamming signal, Huang et al. [18] and Li et al. [19] utilized machine learning to classify the target and jammer, respectively.

While various methods have been investigated to improve the anti-jamming capacity of FMCW sensors, it is of great importance to have a specific method for short-range FMCW proximity sensors. The special operating environment leads to two issues that generally do not exist in conventional radar designs. Due to the signal leakage, close range, and the generally narrow antenna beam, the target is only partially illuminated, leading to a relatively low signal-to-noise rate (SNR) [20,21]. Due to the small volume and power limitations, the hardware is generally simple and cost-effective, resulting in implementation difficulties of the complex circuits and algorithms.

One kind of proximity sensors uses harmonic signal information to detect targets. Through analyzing the spectrum of a short-range FMCW proximity sensor's beat signal, it was found that the spectral harmonic component peaked at some specific distances. Existing sensors usually use BPF to extract the harmonic signal. However, the filter design has a direct effect on the suppression of jamming [22-24]. For instance, when more harmonic signals are used, the BPF-based harmonic acquisition method consumes a larger number of system resources, causing a non-negligible processing delay and performance decrease.

This paper presents an anti-sweep jamming design and its implementation for short-range FMCW proximity sensors using the multi-channel harmonic timing sequence detection method. The harmonic peak amplitude information and timing information of multi-channels are used to suppress the jamming. To reduce the resource consumption, our study implements the harmonic timing detection based on FFT. The multi-channel harmonic envelope information is acquired using FFT once, in each transmission cycle. The main contributions of this paper are as follows: (1) the failure mechanism of the sensor under sweep jamming is analyzed; (2) an anti-jamming method of multi-channel harmonic timing detection is proposed and implemented based on FFT; and (3) the anti-jamming capability is improved and resource usage is reduced.

The rest of the paper is organized as follows: the principles of short-range FMCW proximity sensors, the construction of the sweep jamming model, and the analysis of the theoretical basis of anti-sweep jamming are introduced in Section 2. Section 3 demonstrates the implementation of multi-channel harmonic timing detection based on the BPF and FFT, respectively. The simulation analysis of two harmonic timing detection methods is presented, and the sensor prototype is designed to verify the ranging and anti-jamming capability in Section 4, which is followed by our conclusions in Section 5.

\section{Failure Mechanism Analysis of Short-Range FMCW Proximity Sensor under Sweep Jamming}

\subsection{FMCW Harmonic Ranging Principle}

The FMCW sensor, shown schematically in Figure 1, transmits an FM signal, and mixes the transmitted signal and the target echo signal to obtain the beat signal after low-pass filtering. From the beat signal, the range information can be calculated.

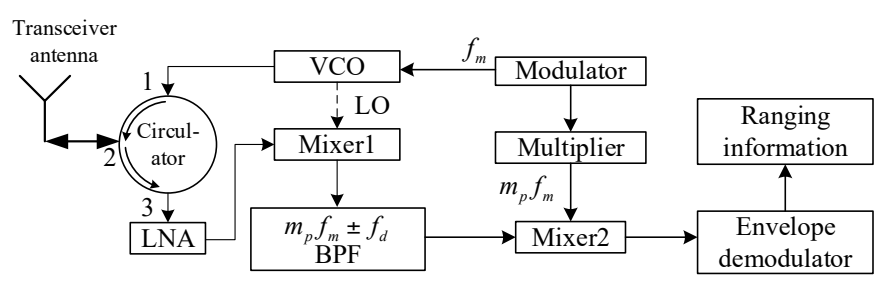

Figure 1. Block diagram of a short-range FMCW proximity sensor (LNA: low-noise amplifier; VCO: voltage-controlled oscillator; LO: local signal). 
The signal from the voltage-controlled oscillator (VCO) (controlled by the modulator) enters Port 1 of the circulator. Almost all of the energy of this signal passes from Port 2 to the transceiver antenna and is radiated toward the target. The expression of the transmission modulated by triangular waveform is

$$
s_{t}(t)= \begin{cases}A_{t} \cos \left\{2 \pi\left[f_{c}+(4 n+1) \Delta F\right] t-\pi \beta t^{2}\right\} & n T \leq t \leq n T+\frac{T}{2} \\ A_{t} \cos \left\{2 \pi\left[f_{c}-(4 n+3) \Delta F\right] t+\pi \beta t^{2}\right\} & n T+\frac{T}{2} \leq t \leq(n+1) T\end{cases}
$$

where $A_{t}$ is the amplitude of the transmitted signal, $f_{c}$ is the carrier frequency, $\Delta F$ is half of bandwidth, $\beta$ is the FM slope, $n$ is the number of transmission, $T$ is the period of modulation signal, and $f_{m}=\frac{1}{T}$ is the frequency of the modulation signal. To ensure the achievable implementation and maximum range detection, $f_{m}$ is set to be $f_{d} \ll f_{m} \ll \frac{c}{4 R_{\max }}$, where $f_{d}$ is the Doppler signal produced by relative motion, $c$ is the speed of light, and $R_{\max }$ is the maximum detection range of the proximity sensors.

The signal reflected is an attenuated and delayed version of the transmitted signal, expressed as $s_{r}(t)=A_{e} \cdot s_{t}(t-\tau)$, where $A_{e}$ is the attenuation factor, and $\tau$ is the round-trip time for the signal to propagate from the sensor system and back. The echo signal enters Port 2 of the circulator, passes to Port 3 and on to mixer1. Another port of mixer1 is connected to the local signal $s_{l}(t)$ with amplitude $A_{l}$. The output of mixer1 (called the beat signal) can be expressed as

$$
s_{b}(t)= \begin{cases}\frac{A_{e} A_{t} A_{l}}{2} \cos \left\{2 \pi \beta t^{2}-2 \pi \beta \tau t-16 \pi n t-2 \pi\left[f_{c}-(4 n-1) \Delta F\right] \tau+\pi \beta \tau^{2}\right\}, & n T \leq t<n T+\tau \\ \frac{A_{e} A_{t} A_{l}}{2} \cos \left\{2 \pi \beta \tau t-2 \pi\left[f_{c}+(4 n+1) \Delta F\right] \tau-\pi \beta \tau^{2}\right\}, & n T+\tau \leq t<n T+\frac{T}{2} \\ \frac{e_{e} A_{t} A_{l} A_{l}}{2} \cos \left\{2 \pi \beta t^{2}-2 \pi \beta \tau t-2 \pi(8 n+4) t+2 \pi\left[f_{c}+(4 n+1) \Delta F\right] \tau+\pi \beta \tau^{2}\right\}, & n T+\frac{T}{2} \leq t<n T+\frac{T}{2}+\tau \\ \frac{A_{e} A_{t} A_{l}}{2} \cos \left\{2 \pi \beta \tau t+2 \pi\left[f_{c}-(4 n+3) \Delta F\right] \tau-\pi \beta \tau^{2}\right\}, & n T+\frac{T}{2}+\tau \leq t<(n+1) T\end{cases}
$$

As $\tau \ll T$ for the proximity sensor [22], the beat signal between $n T \leq t<n T+\tau$ and $n T+\frac{T}{2} \leq t<n T+\frac{T}{2}+\tau$ can be ignored. The spectrum of $s_{b}(t)$ is

$$
F_{b}(f, \tau)=\frac{A_{e} A_{t} A_{l}}{2} \sum k(m, \tau) \delta\left(f-m f_{m} \pm f_{d}\right)
$$

where $m$ is the harmonic number, and

$$
k(m, \tau)=\left\{\begin{array}{l}
\left(1-\frac{2 \tau}{T}\right)\left|\left(\sin c\left[\left(2 \pi \beta \tau-2 \pi m f_{m}\right)\left(\frac{T}{4}-\frac{\tau}{2}\right)\right]+\sin c\left[\left(2 \pi \beta \tau+2 \pi m f_{m}\right)\left(\frac{T}{4}-\frac{\tau}{2}\right)\right]\right) \cos \left(2 \pi f_{c} \tau\right)\right|, m \text { is even } \\
\left(1-\frac{2 \tau}{T}\right)\left|\left(\sin c\left[\left(2 \pi \beta \tau-2 \pi m f_{m}\right)\left(\frac{T}{4}-\frac{\tau}{2}\right)\right]-\sin c\left[\left(2 \pi \beta \tau+2 \pi m f_{m}\right)\left(\frac{T}{4}-\frac{\tau}{2}\right)\right]\right) \sin \left(2 \pi f_{c} \tau\right)\right|, m \text { is odd }
\end{array}\right.
$$

As shown in Equation (3), the spectrum of the beat signal consists of $m f_{m} \pm f_{d}$. The energy of the separate spectrum is described in Equation (4), which is composed of two Sinc function. The $k(m, \tau)$ display peaks when $\left(2 \pi \beta \tau-2 \pi m f_{m}\right)\left(\frac{T}{4}-\frac{\tau}{2}\right)=0$, deriving

$$
m=\frac{8 \Delta F \cdot R}{c}
$$

As shown above, when the predetermined distance $R_{p}$ is given, the corresponding harmonic number $m_{p}$ can be obtained from Equation (5). Hence, in practice, the detection of predetermined distance $R_{p}$ is always converted to the detection of the signal peak of $m_{p}$ harmonic. Generally, $m_{p}$ is designed to be an even number to suppress the damage of the leakage signal [25]. Equation (5) indicates that, when the sensor detects the target and the distance is reduced, the high-to-low-order harmonic signal peaks appear in turn and that the range resolution is $\Delta R=\frac{c}{4 \Delta F}$.

The harmonic signal carrying Doppler information is obtained by BPF. The output of BPF is

$$
s_{b p}(t)=\frac{A_{e} A_{t} A_{l}}{2} k\left(m_{p}, \tau\right) \cos \left[2 \pi\left(m_{p} f_{m} \pm f_{d}\right) t\right]
$$

The $s_{b p}(t)$ is mixed with the reference signal in mixer2. The frequency of reference signal is $m_{p} f_{m}$. The output of mixer2 is passed to the envelope demodulator (shown in Figure 1), and the obtained 
Doppler signal is expressed in Equation (7). The threshold detection is performed using the Doppler signal to provide the ranging information.

$$
s_{d}(t)=\frac{A_{e} A_{t} A_{l}}{2} k\left(m_{p}, \tau\right) \cos \left(2 \pi f_{d} t\right)
$$

\subsection{Sweep Jamming Strategy}

In sweep jamming, the jammer sweeps its frequency from one to the other and does not share its power among multiple frequencies.

The parameters of sweep jamming are as follows: the start frequency $f_{j 0}$, the stop frequency $f_{j N}$, the frequency step $\Delta f_{j}$, the dwell time $T_{d w}$, and the sweep number $N=\left(f_{j N}-f_{j 0}\right) / \Delta f_{j}$. The instantaneous frequency of the sweep jamming signal is

$$
f_{j}(t)=f_{j 0}+\Delta f_{j} \cdot \sum_{k=0}^{N-1} k \cdot P_{T_{d w}}\left(t-\frac{T_{d w}}{2}-k T_{d w}\right)
$$

where $k=0,1, \cdots, N-1$, and $P_{T_{d w w}}(t)=\left\{\begin{array}{cc}1, & |t| \leq T_{d w} / 2 \\ 0, & \text { others }\end{array}\right.$.

The phase of sweep jamming is

$$
\varphi_{j}(t)=2 \pi \int_{0}^{t} f_{j}(t) d t=2 \pi\left[\left(f_{j 0}+k \cdot \Delta f_{j}\right) \cdot t-\frac{k \cdot(k+1)}{2} \Delta f_{j} \cdot T_{d w w}\right]
$$

where $k \cdot T_{d w}<t \leq(k+1) \cdot T_{d w}, \quad k=0,1, \cdots, N-1$.

Sweep jamming is generally used with amplitude modulation (AM), e.g., cos wave amplitude modulation, so the jamming signal $s_{j}(t)$ can be expressed as

$$
s_{j}(t)=\left[A_{j}+A_{j M} \cos \left(2 \pi f_{j M} t+\varphi_{j M}\right)\right] \cos \left(\varphi_{j}(t)\right)
$$

where $A_{j}$ is the amplitude of carrier signal, $A_{j \mathrm{M}}$ is the amplitude of AM signal, $f_{j \mathrm{M}}$ is the frequency of AM signal, and $\varphi_{j \mathrm{M}}$ is the initial phase of the AM signal, assuming $\varphi_{j \mathrm{M}}=0$ here. The amplitude modulation results in the spectrum shifting of $f_{j}(t)$, e.g., at the kth sweep point, and the instantaneous frequency consists of both $f_{j 0}+k \cdot \Delta f_{j} \pm f_{j M}$ and $f_{j 0}+k \cdot \Delta f_{j}$ after $\mathrm{AM}$, as shown in Figure 2 .

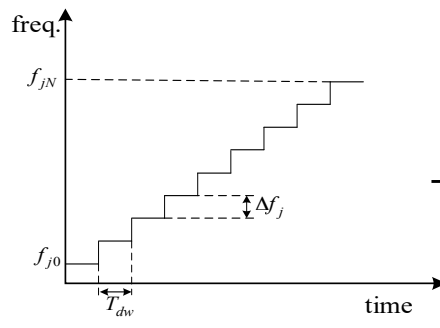

frequency sweep signal

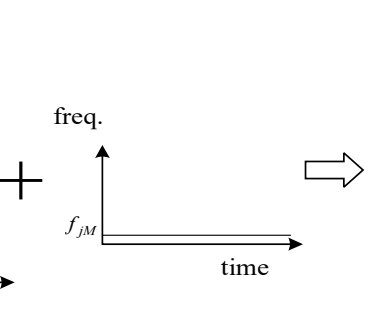

amplitude modulation signal

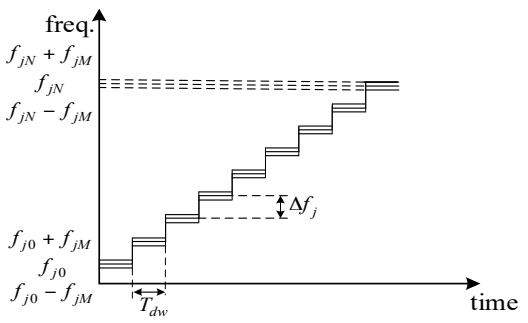

sweep jamming with AM

Figure 2. Block diagram of time-frequency analysis for the sweep jamming signal with AM.

\subsection{Failure Mechanism Analysis}

The sweep jamming signal is received by the sensor transceiver and mixed with the LO; the beat signal under jamming can be expressed as

$$
\begin{aligned}
s_{i j}(t) & =\frac{A_{l}}{2}\left[A_{j}+A_{j M} \cos \left(2 \pi f_{j M} t\right)\right] \cdot \cos \left[\varphi_{j}(t)-\varphi_{l}(t)\right] \\
& =s_{i j 1}(t) \cdot s_{i j 2}(t)
\end{aligned}
$$


where $\varphi_{L}(t)$ is the phase of local signal, and

$$
\begin{aligned}
& s_{i j 1}(t)=\frac{A_{l}}{2}\left[A_{j}+A_{j M} \cos \left(2 \pi f_{j M} t\right)\right] \\
& s_{i j 2}(t)=\cos \left[\varphi_{j}(t)-\varphi_{l}(t)\right]
\end{aligned}
$$

The Fourier transform (FT) of $s_{i j}(t)$ is $S_{i j}(f)$ :

$$
S_{i j}(f)=S_{i j 1}(f) * S_{i j 2}(f)
$$

where $S_{i j 1}(f)$ and $S_{i j 2}(f)$ are the FT of $s_{i j 1}(t)$ and $s_{i j 2}(t)$, respectively. The first item on the right side of Equation (13) shifts the spectrum of $S_{i j 2}(f)$ and simulates the Doppler phenomenon during the encounter. The spectrum of $s_{i j}(t)$ is mainly dependent on $s_{i j 2}(t)$.

$$
s_{i j 2}(t)=\left\{\begin{array}{l}
\cos \left\{\begin{array}{l}
2 \pi\left[\left(f_{c}+(4 n+1) \Delta F-f_{j 0}-k \cdot \Delta f_{j}\right) t\right. \\
\left.-\pi \beta t^{2}+\frac{k \cdot(k+1)}{2} \Delta f_{j} \cdot T_{d w}\right] \\
2 \pi\left[\left(f_{c}-(4 n+3) \Delta F-f_{j 0}-k \cdot \Delta f_{j}\right) t\right. \\
\cos t^{2}+\frac{k \cdot(k+1)}{2} \Delta f_{j} \cdot T_{d w w}
\end{array}\right\}, \quad k \cdot T_{d w w}<n T \leq t \leq n T+\frac{T}{2} \leq(k+1) \cdot T_{d w w} \\
k \cdot T_{d w w}<n T+\frac{T}{2} \leq t \leq(n+1) T \leq(k+1) \cdot T_{d w w}
\end{array}\right.
$$

The dwell time of sweep jamming is in the order of milliseconds, while the FM period is in the order of microseconds. Consequently, the sweep point is considered to be the same in a transceiver cycle. $s_{i j 2}(t)$ can then be expressed as

$$
\begin{gathered}
s_{i j 2}(t)=\cos \left[2 \pi\left(f_{c}-f_{j 0}-k \cdot \Delta f_{j}\right) t\right] \sum_{m=-\infty}^{+\infty} a_{m} e^{j 2 \pi m f_{m} t} \\
a_{m}=\cos \left[\frac{\pi(\mu+m)^{2}}{4 \mu}\right][C(a)+C(b)]-\sin \left[\frac{\pi(\mu+m)^{2}}{4 \mu}\right][S(a)+S(b)]
\end{gathered}
$$

where $C(a), C(b), S(a)$, and $S(b)$ are Fresnel functions, and $\mu=\frac{\Delta F}{f_{m}}, a=\frac{\mu-m}{\sqrt{2 \mu}}, b=\frac{\mu+m}{\sqrt{2 \mu}}$. The spectrum of $s_{i j}(t)$ is composed of $f_{c}-f_{j 0}-k \cdot \Delta f_{j} \pm m f_{m} \pm f_{j M}$, with bandwidth $2 \Delta F$.

The center frequency of sweep jamming is generally equal to the carrier frequency, and sweep bandwidth covers the bandwidth of the sensor. At the beginning of the sweep, the non-cooperative relationship between the sweep jamming and sensor transmission signal keeps the effective jamming signal out of the BPF's passband; hence, the sensor is not jammed. With changes in the sweep frequency step, the effective jamming signal gradually passes through the BPF's passband, that is, the signal appears in $m_{p} f_{m} \pm f_{j M}$. While the jamming signal's energy is large enough, the harmonic energy satisfies the condition for ranging, causing the sensor to be jammed.

When the sensor receives the sweep jamming signal, the corresponding peaks appear randomly in a certain number of harmonics, determined by the jamming parameters. However, when the sensor detects the target and its distance is reduced, the high-to-low-order harmonic signal peaks appear in a regular sequence. Therefore, harmonic peak timing sequence detection can be used to differentiate between the target echo and jamming signal.

\section{Design and Implementation of Multi-Channel Harmonic Timing Sequence Detection}

\subsection{Multi-Channel Harmonic Timing Sequence Detection Based on BPF}

The block diagram of the designed method based on BPF is shown in Figure 3. 


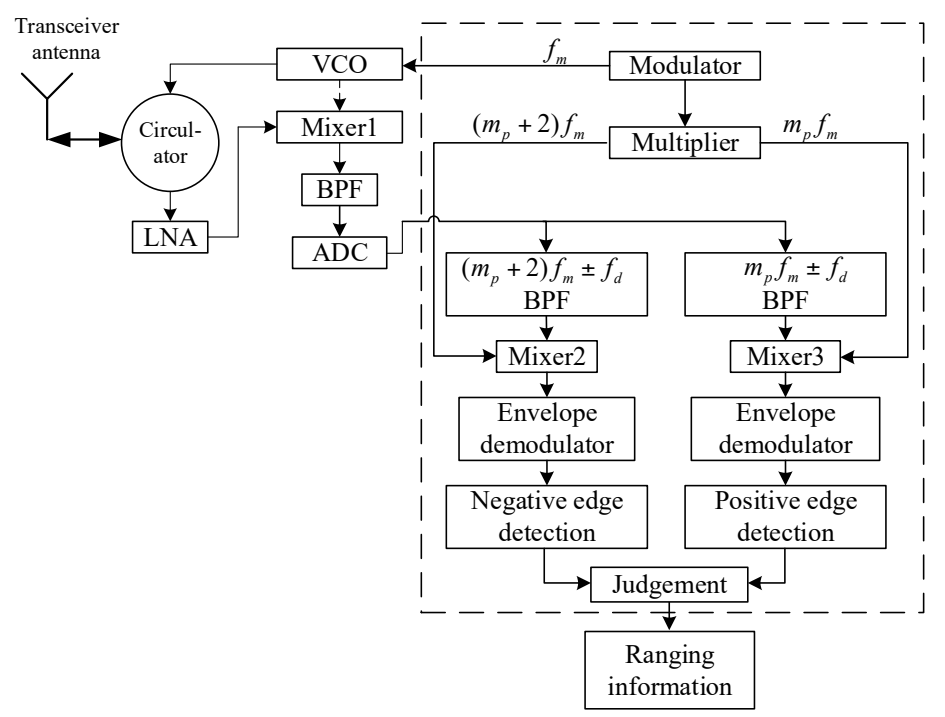

Figure 3. Block diagram of multi-harmonic timing sequence detection based on BPF.

Unlike the scheme in Figure 1, the newly designed sensor comprehensively combined dual-channel information to acquire the predetermined distance $R_{p}$. The negative edge of the $\left(m_{p}+2\right) f_{m} \pm f_{d}$ channel and the positive edge of $m_{p} f_{m} \pm f_{d}$ channel were detected simultaneously. Only when the negative edge comes earlier than the positive edge is the ranging condition satisfied.

Under the condition of sweep jamming, the spectrum of the beat signal is composed of $f_{c}-f_{j 0}-k \cdot \Delta f_{j} \pm m f_{m} \pm f_{j M}$, the valid signal can pass through a dual-channel passband, but the amplitudes of both channels appear randomly during the dwell time of sweep jamming; when the point of sweep frequency comes, the positive edge and negative edge appear simultaneously, resulting in a mismatch of harmonic timing sequence detection.

More channels mean more obvious difference in timing sequence among them, which provides a more rigorous judging condition and better anti-jamming capability. However, this causes an implementation problem at the same time. Regardless of whether the analog filter group or digital filter is utilized, extra system resource consumption will be inevitable when more channels are employed, even though some signal processors such as the Field-Programmable Gate Array (FPGA) provide a special IP core. A larger number of system resources are consumed when the filters' orders are relatively high, causing a non-negligible processing delay and performance decrease. To overcome this disadvantage, the anti-sweep jamming sensor based on FFT was proposed and designed.

\subsection{Multi-Channel Harmonic Timing Sequence Detection Based on FFT}

The block diagram of the designed sensor based on FFT is shown in Figure 4.

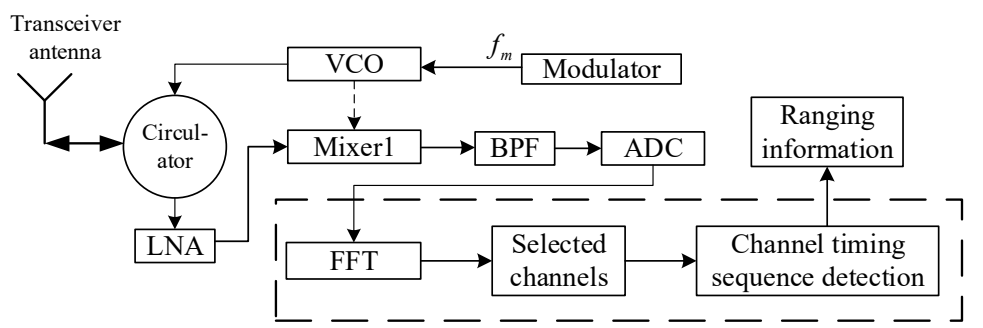

Figure 4. Block diagram of multi-harmonic timing sequence detection based on FFT (The operations in the dashed box are implemented using a digital device). 
Different from the FFT-based instantaneous frequency estimation sensor, the designed sensor, instead of using instantaneous frequency, uses separate components of the beat signal spectrum to achieve harmonic timing sequence detection. The beat signal is sampled by the analog-to-digital converter (ADC) and then transformed using the FFT in the digital signal processor, and the multi-channel signal (changing with range) can be obtained as shown in Figure 5. When the amplitude conditions and timing sequence detection are met, the processor outputs the ranging information.

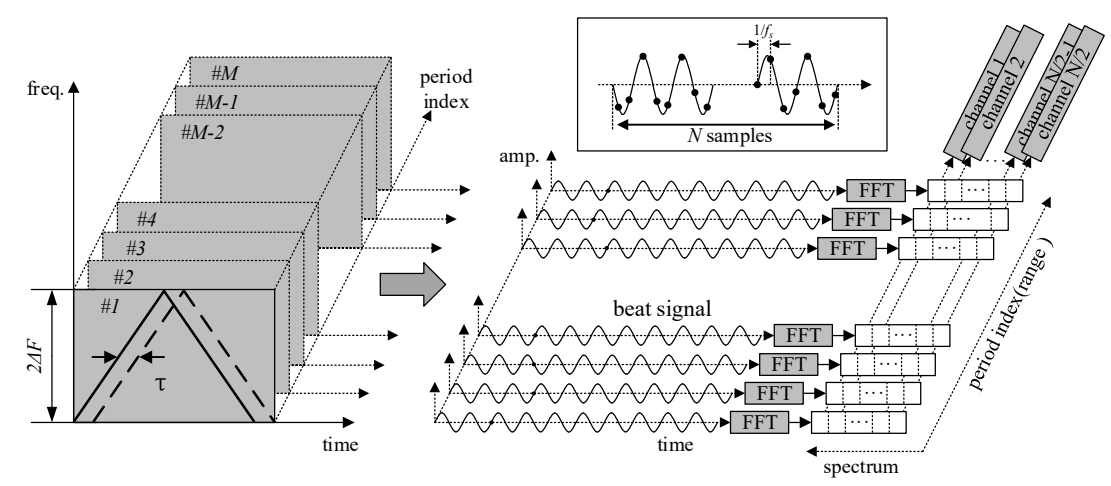

Figure 5. Basic concept of FFT-based harmonic timing sequence detection.

For the anti-sweep jamming sensor based on FFT, FFT is performed once during each transmission cycle to obtain the multi-channel harmonic amplitude. Furthermore, as computational complexity is related to the number of FFT points, the amount of digital resources can be significantly reduced by using this implementation method.

\section{Simulated and Measured Results Discussion}

We used numerical simulations and experimental tests to verify the key performances of the designed sensor: reliable ranging performance and improved anti-sweep jamming performance. In the first scenario, to test the ranging performance, only the target signal existed during the simulation. In the second scenario, the hardware resource usage of the proposed methods (based on the digital BPF implementation and the FFT implementation) were studied. In the third scenario, the outputs of the multi-channels were obtained under sweep jamming by the simulations and prototype experiments. The proposed multi-channel harmonic timing sequence detection was used to range. After numerous Monte Carlo simulations and experimental tests, the corresponding anti-jamming success rates were collected to quantitate the anti-sweep jamming performance.

\subsection{Ranging Performance Simulation and Analysis}

The ranging performance of the BPF and FFT implementation scheme (based on the timing sequence detection method) were simulated when the proximity sensor approached the target. The simulation parameters are illustrated in Table 1. 
Table 1. Simulation parameters.

\begin{tabular}{cc}
\hline Parameters & Value \\
\hline Carrier frequency & X-band \\
FM frequency $f_{m} / \mathrm{KHz}$ & 100 \\
Bandwidth of sensor $2 \Delta F / \mathrm{MHz}$ & 100 \\
Detection range $/ \mathrm{m}$ & $1-15$ \\
Target speed $/(\mathrm{m} / \mathrm{s})$ & 500 \\
Sensor sampling frequency $/ \mathrm{MHz}$ & 5 \\
FFT points & 128 \\
SNR $/ \mathrm{dB}$ & -10 \\
\hline
\end{tabular}

As the proximity sensor works on the condition of low SNR, the background noise of additive white Gaussian noise was added in the simulation and the SNR was $-10 \mathrm{~dB}$. The outputs of the 2th/4th/6th/8th harmonic channels of the BPF-based and FFT-based realization scheme are shown in Figures $6 \mathrm{a}, \mathrm{b}$ and 7 .

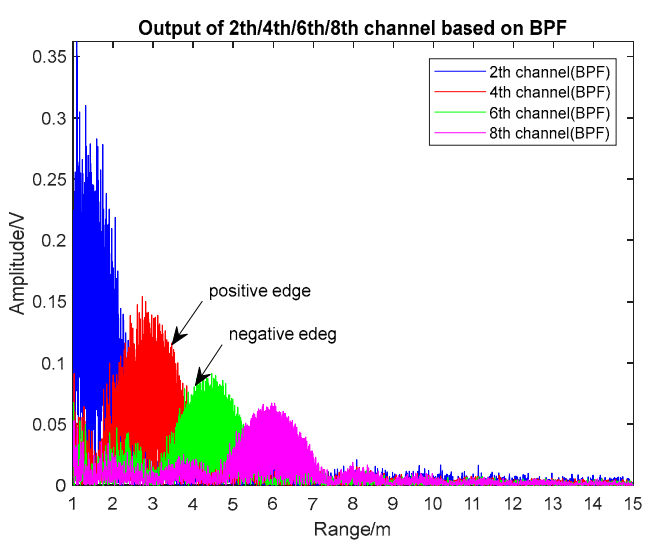

(a)

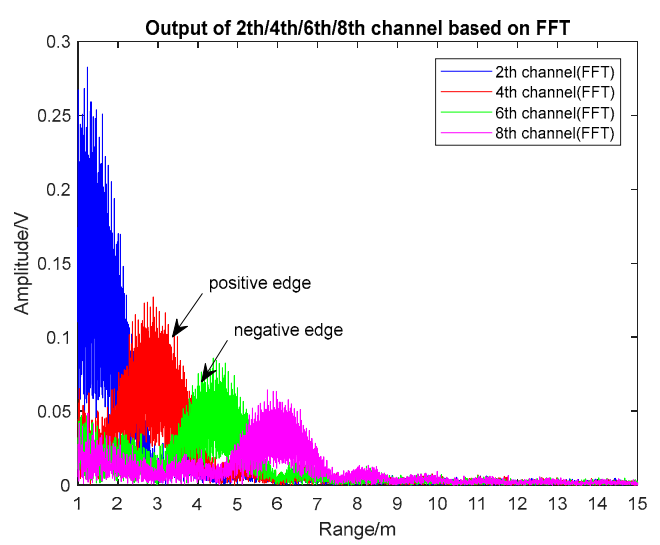

(b)

Figure 6. The outputs of the 2 th $/ 4$ th $/ 6$ th $/ 8$ th harmonic channels (SNR $=-10 \mathrm{~dB}$ ). (a) The BPF-based method, and (b) the FFT-based method.

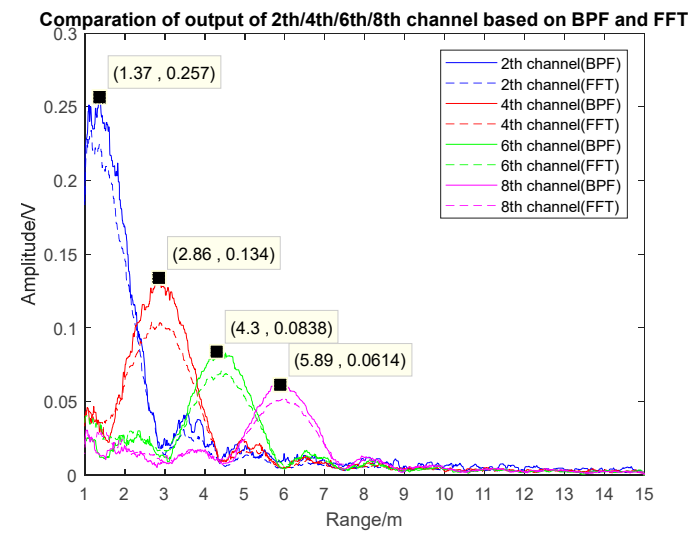

Figure 7. A comparison of the 2th/4th/6th/8th harmonic channels based on the BPF and FFT scheme $(\mathrm{SNR}=-10 \mathrm{~dB})$.

As shown in Figure 6, when the sensor gradually approached the target, the envelope peak of the 8 th $/ 6$ th $/ 4$ th $/ 2$ th harmonic signals appeared successively, and the envelope peak of different harmonic signals increased; namely, the peak of the 2nd harmonic was higher than that of the other 
three channels. Between the two adjacent signal peaks, the high-order harmonic signal was on the negative edge and the low-order harmonic signal was on the positive edge; thus, harmonic timing detection was achieved when a negative edge was followed by a positive edge.

Figure 7 demonstrates that the harmonic envelope based on FFT was basically consistent with the one based on BPF, and the peak points were close to the theoretical points (theoretical peak locations: $1.5 \mathrm{~m}, 3 \mathrm{~m}, 4.5 \mathrm{~m}$, and $6 \mathrm{~m}$, respectively). The maximum ranging error was $0.2 \mathrm{~m}$, which was caused by the increase in echo power when the distance decreased. The range resolution of both methods was $1.5 \mathrm{~m}$, which matched the theoretical value. In the premise of obtained multi-channel harmonic envelopes, timing sequence detection could be achieved through detecting the positive and negative edges.

\subsection{Implementation Complexity Analysis}

The short-range FMCW proximity sensor prototype was designed based on the structure described in Figure 4. Images of the prototype and the RF module are provided in Figure 8a,b.

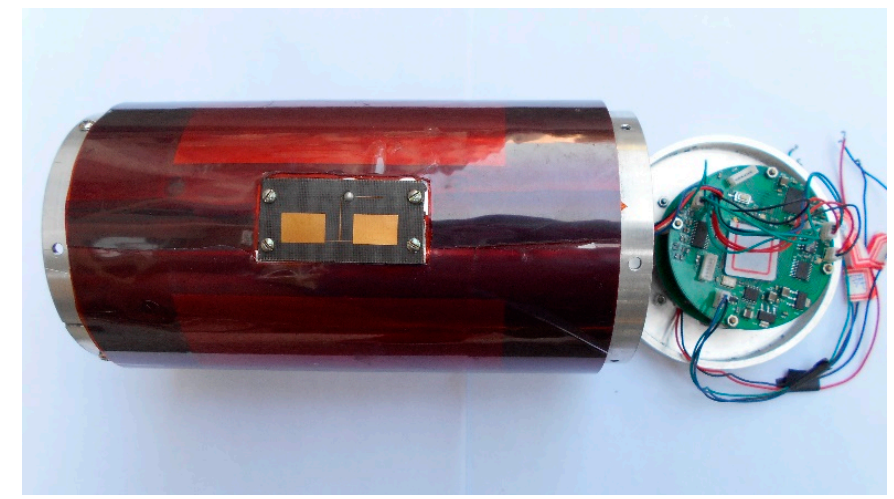

(a)

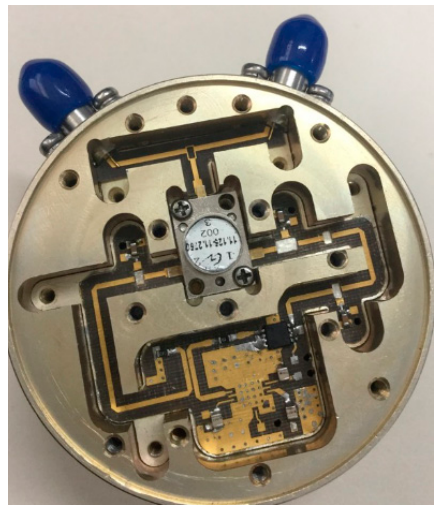

(b)

Figure 8. The designed anti-jamming sensor prototype. (a) The transceiver antenna and signal processing module, and (b) the RF module.

The sub-parts of the RF module were designed using the sub-cavity to reduce the RF signal leakage, and signal processing was completed using the Field-Programmable Gate Array (FPGA). BPF- and FFT-based harmonic timing detection using three channels were implemented on the same hardware, and a comparison of the resources used is shown in Table 2.

Table 2. Implementation complexity analysis (XC3S1000).

\begin{tabular}{cccc}
\hline & \multicolumn{3}{c}{ Parameters } \\
\hline & Number of Slices Flip & Number of 4 input & Number of MULT18X18s \\
BPF & Flops & look-up tabels (LUTs) & 21 \\
FFT & 5256 & 4573 & 8 \\
\hline
\end{tabular}

The implementation of an anti-sweep jamming sensor based on FFT reduced the resource usage significantly; in particular, the number of multipliers used was about $38 \%$ of the number that the BPF-based sensor used. When using the FPGA for FFT with the system clock at $50 \mathrm{MHz}$, and 128 points, FFT takes time at the $\mu$ s level, which satisfies the proximity sensor real-time requirements. To further reduce resource consumption, a harmonic signals envelope could be extracted using data collected on a single up-slope or down-slope, which has been verified. 
The maximum detection range was considered in the design of the RF and signal processing modules. The output power of the transmitted signal was $13 \mathrm{dBm}$ and the transceiver antenna gain was $4 \mathrm{~dB}$. The echo was passed to LNA to ensure that the output of mixer1 was in the order of the $\mathrm{mV}$ level. A 12-bit ADC (resolution 1000/4096 mV) was used to sample the beat signal. The dynamic range was tested and the results met the design specification.

\subsection{Anti-Jamming Test and Analysis}

The anti-jamming performance of the designed sensor was verified by simulation and jamming experiments. The outputs of the $4 \mathrm{th} / 6 \mathrm{th} / 8$ th channels of the sensor under real target echo signals in low-speed encounters (shown in Figure 9) are shown in Figure 10a, and the outputs of the 4th/6th/8th channels of the sensor under sweep jamming are shown in Figure 10b.

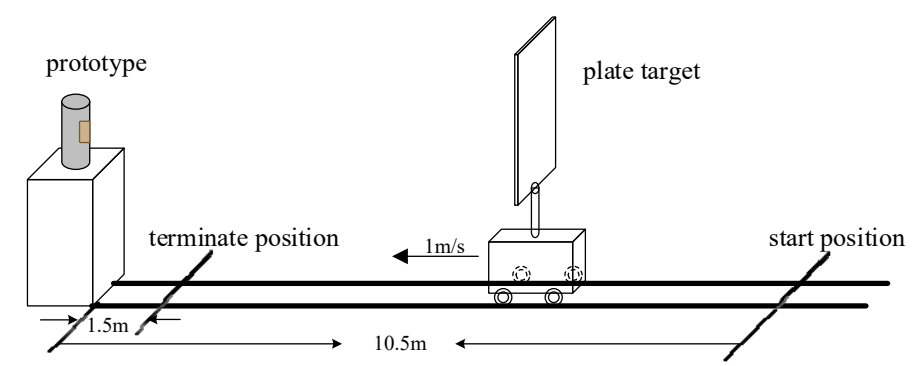

Figure 9. The scenario of a low speed encounter for prototype in the chamber (the metal plate target was fixed on the moving car vertically, and the prototype was placed at one end of the track, loaded by the car moving along the track from 10.5 to $1.5 \mathrm{~m}$ at a speed of $1 \mathrm{~m} / \mathrm{s}$ ).
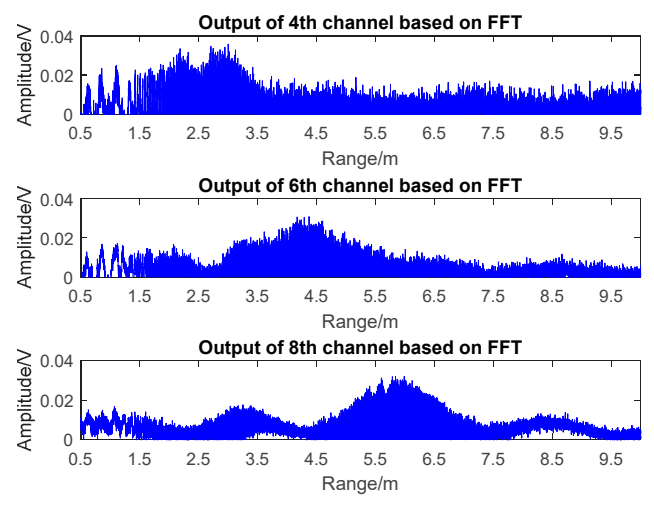

(a)
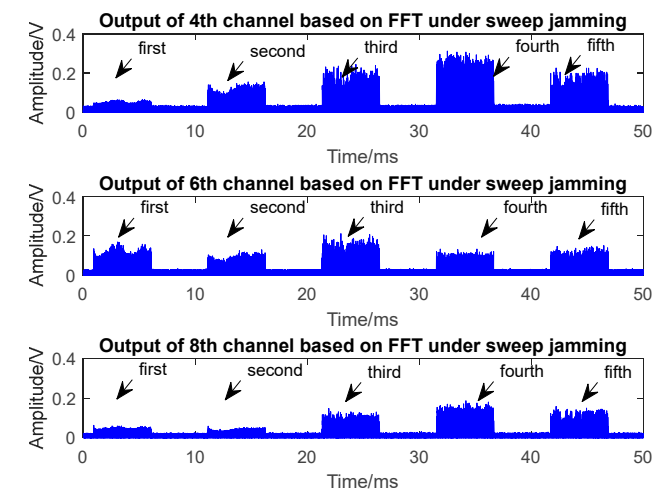

(b)

Figure 10. The 4th/6th/8th channel outputs of the prototype. (a) Low speed encounter; (b) under sweep jamming. (Sweep jamming parameters: $f_{j N}-f_{j 0}=100 \mathrm{MHz} ; \Delta f_{j}=500 \mathrm{KHz} ; T_{d w}=4 \mathrm{~ms}$; AM signal: sine; $f_{j \mathrm{M}}=1 \mathrm{KHz}$.)

Figure 10a shows that the 8th harmonic peak at $6 \mathrm{~m}$, the 6 th harmonic peak at $4.5 \mathrm{~m}$, the 4 th harmonic peak at $3 \mathrm{~m}$, and the peaks of the $8 \mathrm{th} / 6 \mathrm{th} / 4$ th harmonic signals appear in turn. Considering the practical experiment process where the car began to slow down at a distance of $2 \mathrm{~m}$ from the prototype and finally stopped at $1.5 \mathrm{~m}$, the irregular signal at range $1.5-2 \mathrm{~m}$ (caused by braking process of the plate target) was normal and did not lead to a false alarm by the sensor.

The output of the 8th/6th/4th harmonic signals under sweep jamming in Figure 10b was achieved under the condition that the effective jamming signal passed through the BPF's passband. The update period of the sweeping jammer was $6 \mathrm{~ms}$ according to its technical documents. As long as the 4 th channel appeared to be jammed, the other two channels were jammed too, as the spectrum of the beat 
signal is composed of $f_{c}-f_{j 0}-k \cdot \Delta f_{j} \pm m f_{m} \pm f_{j M}$. However, the amplitudes of the three channels appeared randomly during one dwell time in the whole sweep jamming cycle. At the first sweep point, the output of the 6th channel was higher than that of the 8th and 4th channels; at the fourth sweep point, the output of the 8th channel was lower than that of the 4th channel, but was higher than the output of the 6th channel; at the second, third, and fifth sweep point, the variation of the output signal amplitude of the three channels satisfied the amplitude change under the target echo, but as the point of sweep frequency changed, the positive edge and negative edge appeared simultaneously. This was essentially different from the outputs of the multi-channels under the real target echo signal and resulted in a mismatch of harmonic timing sequence detection.

Two thousand simulation experiments using the Monte Carlo method were also conducted. The simulated jamming covered sweep jamming, sweep jamming with AM, and sweep jamming with FM. The AM/FM waveforms consisted of a positive ramp, negative ramp, sin, triangle, square, Gaussian noise, and exponential function. The simulation results showed that the anti-jamming success rate of the designed sensor was $95.5 \%$. With the same jamming parameters, the prototype and the sensor using the structure described in Figure 1 were carried out with the anti-jamming experiment 1000 times, and the success rate of the anti-jamming was $91.3 \%$ and $16.7 \%$, respectively. The simulation and anti-jamming experiment results showed that the proposed method improved the anti-sweep jamming capability of the FMCW sensor effectively.

\section{Conclusions}

To overcome the sweep jamming problem faced by the FMCW proximity sensor in a jamming environment, this study designed a short-range FMCW proximity sensor using harmonic timing sequence extraction. Based on an analysis of the failure mechanisms of a proximity sensor under sweep jamming, we proposed an anti-jamming method of multi-channel harmonic timing sequence detection. To consume fewer hardware resources, FFT-based implementation was employed, and a prototype was designed to verify the effectiveness of the method and the improvements in anti-sweep performance. The simulation and experimental results demonstrated that the sensor described in this paper can effectively improve anti-sweep jamming performance under the condition of the same ranging precision $\Delta R=\frac{c}{4 \Delta F}$.

Acknowledgments: This work is supported by the State Key Program of Basic Research of China under Grant No. 613196 and the National Natural Science Foundation of China under Grant No. 61673066.

Author Contributions: Zhijie Kong designed and implemented the experiments and contributed to analysis and to the writing of the sections related to that analysis. Ping Li and Xiaopeng Yan provided many useful comments and constructive criticism. Xinhong Hao conceived of the idea of the entire study and contributed to the drafting and implementing of the manuscript. All authors read and approved the final manuscript.

Conflicts of Interest: The authors declare no conflict of interest.

\section{References}

1. Li, C.; Peng, Z.; Huang, T.Y.; Fan, T.; Wang, F.K.; Horng, T.S.; Muñoz-Ferreras, J.-M.; Gómez-García, R.; Ran, L.; Lin, J. A Review on Recent Progress of Portable Short-Range Noncontact Microwave Radar Systems. IEEE Trans. Microw. Theory Tech. 2017, 65, 1692-1706. [CrossRef]

2. Li, C.L.; Chen, W.; Liu, G.; Yan, R.; Xu, H.; Qi, Y. A Noncontact FMCW Radar Sensor for Displacement Measurement in Structural Health Monitoring. Sensors 2015, 15, 7412-7433. [CrossRef] [PubMed]

3. Ritchie, M.; Ash, M.; Chen, Q.; Chetty, K. Through Wall Radar Classification of Human Micro-Doppler Using Singular Value Decomposition Analysis. Sensors 2016, 16, 1401. [CrossRef] [PubMed]

4. Damien, V.; Paul, C.; Roland, C. Localization and Mapping Using Only a Rotating FMCW Radar Sensor. Sensors 2013, 13, 4527-4552. [CrossRef]

5. Yue, K.; Hao, X.; Li, P. An LFMCW detector with new structure and FRFT based differential distance estimation method. Springer Plus 2016, 5, 922. [CrossRef] [PubMed] 
6. Yan, J.B.; Alvestegui, G.G.; Mcdaniel, J.W.; Li, Y.; Gogineni, S.; Rodriguez-Morales, F.; Brozena, J.; Leuschen, C.J. Ultrawideband FMCW Radar for Airborne Measurements of Snow Over Sea Ice and Land. IEEE Trans. Geosci. Remote Sens. 2017, 55, 834-843. [CrossRef]

7. Choi, J.H.; Jang, J.H.; Huang, T.Y.; Fan, T.; Wang, F.K.; Horng, T.S.; Muñoz-Ferreras, J.-M.; Gómez-García, R.; Ran, L.; Lin, J. Design of an FMCW radar altimeter for wide-range and low measurement error. IEEE Trans. Instrum. Meas. 2015, 64, 3517-3525. [CrossRef]

8. Balal, N.; Pinhasi, G.A.; Pinhasi, Y. Atmospheric and Fog Effects on Ultra-Wide Band Radar Operating at Extremely High Frequencies. Sensors 2016, 16, 751. [CrossRef] [PubMed]

9. Wang, R.; Chen, J.; Wang, X.; Sun, B. High-Performance Anti-Retransmission Deception Jamming Utilizing Range Direction Multiple Input and Multiple Output (MIMO) Synthetic Aperture Radar (SAR). Sensors 2017, 17, 123. [CrossRef] [PubMed]

10. Choi, Y.S.; Lee, S.Y.; Choi, H.H.; Lee, S.J.; Park, C.S. The improved spatial nuller with frequency swept jammer. In Proceedings of the Position, Location and Navigation Symposium, Monterey, CA, USA, 5-8 May 2014; pp. 1084-1087.

11. Choi, D.Y.; Kim, W.K.; Kim, J.H.; Cho, H. Performance of analog and digital modulation schemes under sweep jamming. In Proceedings of the Eighth International Conference on Ubiquitous and Future Networks, Vienna, Austria, 5-8 July 2016; pp. 13-15.

12. Mighani, S.; Mivehchy, M.; Sabahi, M.F. Evaluating sweep noisy barrage jamming effect on tracking radar based on functioning destruction time. In Proceedings of the International Symposium on Telecommunications, Tehran, Iran, 9-11 September 2014; pp. 400-404.

13. Choi, J.H.; Lee, J.M.; Jung, M.S.; An, J.Y.; Kim, K.L. FMCW transceiver of short-range proximity sensor in sea clutter. Microw. Opt. Technol. Lett. 2017, 59, 334-337. [CrossRef]

14. Choi, J.H.; Jung, M.S.; Yeom, K.W. A design and assessment of a direction finding proximity fuze sensor. IEEE Sens. J. 2013, 13, 3079-3089. [CrossRef]

15. You, P.; Liu, Z.; Wang, H.; Wei, X.; Li, X. Dynamic compressed HRRP generation for random stepped-frequency radar based on complex-valued fast sequential homotopy. Sensors 2014, 14, 8283-8304. [CrossRef] [PubMed]

16. Max, P. New Generation Naval Artillery Multi-Function Fuze. In Proceedings of the 56th Annual Fuze Conference, Baltimore, MD, USA, 12-15 May 2012; pp. 1-20.

17. Xiao, Z.; Zhang, H. Research on Modeling and Simulation of Echo Signal of Pulse Doppler Fuze and Judgment Criterion of Its Impact. Acta Armament. 2016, 37, 1820-1827.

18. Huang, Y.; Hao, X.; Kong, Z.; Zhang, B. Recognition of target and jamming signal for FM fuze based on entropy features. Acta Armament. 2017, 38, 254-260.

19. Li, Z.; Hao, X.; Chen, H.; Li, P. Target signal recognition for CW Doppler proximity radio detector based on SVM. In Proceedings of the International Conference on Mechatronic Sciences, Electric Engineering and Computer, Shengyang, China, 20-22 December 2013; pp. 1160-1163.

20. Beasley, P. The Influence of Transmitter Phase Noise on FMCW Radar Performance. In Proceedings of the Radar Conference, Manchester, UK, 13-15 September 2006; pp. 331-334.

21. Fericean, S.; Dorneich, A.; Droxler, R.; Krater, D. Development of a Microwave Proximity Sensor for Industrial Applications. IEEE Sens. J. 2008, 9, 870-876. [CrossRef]

22. Komarov, I.; Sergey, M. Fundamentals of Short-Range FM Proximity Sensor; Artech House: London, UK, 2003; pp. 111-112.

23. Zuo, H.Y.; Hao, X.H.; Yue, K. Anti-AM Jamming Performance of FM Doppler Fuze. Available online: http:/ / www.cnki.net/kcms/detail/11.2625.V.20170203.1448.001.html (accessed on 20 July 2017).

24. Ayhan, S.; Scherr, S.; Bhutani, A.; Fischbach, B.; Pauli, M.; Zwick, T. Impact of frequency ramp nonlinearity, phase noise, and SNR on FMCW radar accuracy. IEEE Trans. Microw. Theory Tech. 2016, 64, 3290-3301. [CrossRef]

25. Zhao, H.C. Fundamentals and Methodology of Radio Fuze; National Defense Industry Press: Beijing, China, 2012; pp. 55-56.

(C) 2017 by the authors. Licensee MDPI, Basel, Switzerland. This article is an open access article distributed under the terms and conditions of the Creative Commons Attribution (CC BY) license (http:/ / creativecommons.org/licenses/by/4.0/). 